\title{
CRESCIMENTO E SOBREVIVÊNCIA DE JUVENIS DO LINGUADO Paralichthys orbignyanus: EFEITOS DO ENRIQUECIMENTO DA ARTEMIA SP. COM N-3 HUFA
}

RICARDO VIEIRA RODRIGUES ${ }^{1}$, LUCIANO SIQUEIRA FREITAS ${ }^{1}$, RICARDO BERTEAUX ROBALDO² E LUÍS ANDRÉ SAMPAIO ${ }^{1}$ 'Universidade Federal do Rio Grande - Instituto de Oceanografia, Estação Marinha de Aquacultura, Rua do Hotel 2, Cassino, Rio Grande - RS - Brasil. CEP: 96210-030 - vr.ricardo@gmail.com

${ }^{2}$ Universidade Federal de Pelotas - UFPEL, Instituto de Biologia, Departamento de Fisiologia e Farmacologia, CP 354, Pelotas - RS, CEP 96010-900, Brasil

\begin{abstract}
O linguado Paralichthys orbignyanus é um forte candidato para a aquicultura na América do Sul e a produção de juvenis é uma das etapas cruciais para a sua produção em larga escala. Sabe-se que o enriquecimento da Artemia com ácidos graxos altamente insaturados ( $n-3$ HUFA) pode otimizar a produção de muitos peixes marinhos. Portanto, o objetivo deste trabalho foi verificar os benefícios do enriquecimento da Artemia com n-3 HUFA para a produção de juvenis de $P$. orbignyanus. Juvenis recém-assentados de linguado foram transferidos para seis unidades experimentais, onde foram alimentados com náuplios de Artemia ou Artemia enriquecida. Os peixes foram alimentados ad libitum à temperatura e salinidade de $24^{\circ} \mathrm{C}$ e 32 , respectivamente. Os linguados alimentados com Artemia enriquecida cresceram significativamente mais e tiveram maior sobrevivência $(P<0,05)$ que os linguados alimentados com Artemia não enriquecida. Contudo, a taxa de pigmentação foi similar entre linguados alimentados com Artemia enriquecida e não enriquecida. Conclui-se que a produção de juvenis de $P$. orbignyanus pode ser otimizada através do enriquecimento da Artemia com emulsões comerciais ricas em n-3 HUFA.
\end{abstract}

RESUMO

PALAVRAS CHAVE: piscicultura marinha, ácidos graxos, linguado

\section{ABSTRACT \\ Growth and survival of juvenile Brazilian flounder Paralichthys orbignyanus: effects of N-3 HUFA Artemia sp. enrichment}

The Brazilian flounder Paralichthys orbignyanus is a candidate for aquaculture in South America and the healthy juvenile production is the major bottleneck for its mass production. Enrichment of Artemia with highly unsaturated fatty acids ( $n-3$ HUFA) improves the output of most marine fish larviculture. Therefore, the aim of this work was to verify the benefits of using enriched Artemia on the production of $P$. orbignyanus. Early juvenile flounder were transferred from a larviculture tank to six experimental units, where they were fed either enriched or non-enriched Artemia. Fish were fed ad libitum at temperature and salinity $24^{\circ} \mathrm{C}$ and 32, respectively. Furthermore, flounder fed enriched Artemia had significantly $(P<0.05)$ higher survival and growth. However, the percentage of well pigmented flounder was similar when they were fed Artemia nauplii or enriched Artemia. In conclusion, the production of Brazilian flounder juveniles can be improved through the enrichment of Artemia with commercial emulsions of $n-3$ HUFA.

KEYWORDS: marine fish culture, fatty acids, flatfish

\section{INTRODUÇÃO}

O linguado Paralichthys orbignyanus é encontrado ao longo de águas costeiras e estuarinas do Brasil, Uruguai e Argentina (Lema et al. 1980), sendo um importante recurso pesqueiro nestas áreas. Esta espécie tem recebido atenção para o desenvolvimento da aquicultura no extremo sul do Brasil, nos últimos anos, especialmente pelo seu elevado valor de mercado e tolerância a parâmetros físicos e químicos, como compostos nitrogenados (Bianchini et al. 1996), pH (Wasielesky et al. 1997), temperatura (Wasielesky et al. 1998) e salinidade (Sampaio et al. 2001; Sampaio \& Bianchini 2002; Sampaio et al. 2007). O desenvolvimento da metodologia para a produção de juvenis vem sendo aprimorada (Sampaio et al., 2008; Bianchini et al., 2010), mas algumas questões ainda são limitantes, entre elas a alimentação e nutrição de larvas e juvenis.

Watanabe et al. (1983) relataram que durante os primeiros estádios de desenvolvimento dos peixes, mortalidades elevadas ou taxas de crescimento reduzidas podem ocorrer, normalmente relacionadas a uma nutrição inadequada. Deficiências nutricionais também podem causar a problemas de pigmentação em juvenis de linguado (Venizelos \& Benetti 1999).

Estudos sobre a qualidade nutricional do alimento vivo demonstraram a importância dos ácidos graxos altamente insaturados ( $n-3$ HUFA), para o desenvolvimento de larvas de peixes marinhos, pois estes ácidos graxos são considerados essenciais (AGE) para estas larvas (Watanabe et al. 1983; Léger et al. 1986). Como os peixes marinhos possuem limitada capacidade de sintetizar estes ácidos graxos "de novo" (Kanazawa 1997), torna-se necessário adicionar ao alimento algum ingrediente que seja rico nesses ácidos graxos. Os AGE são parte importante de sua dieta, pois eles são os maiores componentes dos fosfolipídeos das membranas celulares e das membranas do sistema nervoso, sendo muito importantes para o crescimento e o desenvolvimento normal dos peixes (Sargent et al. 1995).

Náuplios de Artemia são amplamente utilizados na aquicultura mundial como alimento vivo para a 
produção de peixes marinhos. Contudo, um dos maiores problemas com a utilização da Artemia é seu reduzido valor nutricional, especialmente em relação aos ácidos graxos altamente insaturados, como o ácido eicosapentaenóico (EPA) e o ácido docosahexaenóico (DHA) (Ando et al. 2004). Estes ácidos graxos são indicadores da qualidade nutricional da Artemia (Sorgeloos et al. 1998). Entretanto, de acordo com a sua origem geográfica a concentração desses ácidos graxos nos náuplios de Artemia pode ser baixa ou extremamente variável (Léger et al. 1986; Sorgeloos et al. 2001). Para resolver este problema, métodos para 0 enriquecimento da Artemia com HUFA têm sido desenvolvidos, entre eles, o uso de microalgas, produtos micro-encapsulados, fermento, preparações emulsificadas e produtos micro-particulados. Contudo, os maiores níveis de enriquecimento da Artemia com n-3 HUFA são obtidos com a utilização de produtos emulsificados (Sorgeloos et al. 2001).

Para diferentes espécies de linguado, tais como Paralichthys dentatus, Psetta maxima e Solea senegalensis, o enriquecimento do alimento vivo com n-3 HUFA proporcionou efeitos positivos sobre a sobrevivência, o crescimento e a pigmentação (Reitan et al. 1994; Baker et al. 1998; Morais et al. 2004).

O objetivo do presente trabalho foi determinar os efeitos do enriquecimento da Artemia com n-3 HUFA sobre o crescimento, a sobrevivência, e a pigmentação dos juvenis do linguado $P$. orbignyanus.

\section{MATERIAIS E MÉTODOS}

Reprodutores de $P$. orbignyanus foram capturados na Praia do Cassino, no extremo sul do Brasil (Rio Grande - RS) e transportados para o Laboratório de Piscicultura Estuarina e Marinha da Universidade Federal do Rio Grande - FURG. A reprodução artificial foi realizada através da metologia descrita por Sampaio et al. (2008).

As larvas foram criadas, desde a eclosão, em um tanque cilíndrico de $200 \mathrm{~L}$, com uma densidade de 30 larvas/L, iluminação constante e intensidade lumidade de 100 lux. A temperatura da água do tanque e a salinidade foram mantidas em $24^{\circ} \mathrm{C}$ e 32 , respectivamente. A primeira alimentação, fornecida três dias após eclosão (DAE), foi constituída de rotíferos Brachionus plicatilis (10-20 rotíferos $/ \mathrm{mL}$ ) até - 14- DAE. Durante o período de alimentação com rotíferos, foi utilizado a técnica da "água verde", obtida através da adição da microalga Nannochloropsis oculata, numa concentração de 500.000 células $/ \mathrm{mL}$. Após quinze DAE teve início o período de co-alimentação das larvas, com a utilização de rotífero e náuplios de Artemia recémeclodidos. Este período de transição teve duração de quatro dias, quando então, as larvas foram alimentadas exclusivamente com Artemia.

No vigésimo DAE todas as larvas completaram 0 assentamento. A partir desse dia, os juvenis $(n=$ 70) foram individualmente contados e transferidos para suas respectivas unidades experimentais, compostas por seis tanques de $15 \mathrm{~L}$, sob iluminação constante. A temperatura da água dos tanques foi mantida em $24 \pm 0,5^{\circ} \mathrm{C}$, com auxílio de um sistema de aquecimento constituído de aquecedores (VisiTherm $^{\circledR}$, Modelo Deluxe) e a salinidade foi mantida em 32 .

A alimentação foi fornecida duas vezes ao dia. Foram testados dois tratamentos (juvenis de linguado alimentados com náuplios de Artemia recémeclodidos e juvenis de linguado alimentados com meta-náuplios de Artemia enriquecidos com n-3 HUFA), com três repetições cada.

Biometrias para a verificação do comprimento total e do peso dos linguados realizadas a cada 10 dias. Para as biometrias, os linguados foram previamente anestesiados com benzocaína (50 ppm). Após a anestesia, foram medidos com auxílio de um ictiômetro e pesados em balança (Sartorius, Modelo AC 210 S), com precisão de $1 \mathrm{mg}$.

Os rotíferos $B$. plicatilis foram cultivados em tanques de fibra cilíndrico-cônicos de $150 \mathrm{~L}$, com água a $28^{\circ} \mathrm{C}$, salinidade 25 , iluminação e aeração constantes. A densidade máxima de estocagem foi de 800 rotíferos $/ \mathrm{mL}$. Durante o cultivo eles foram alimentados seis vezes ao dia com Selco 3000 $\left(\right.$ Inve $\left.^{\circledR}\right)$ na proporção de $0,4 \mathrm{~g}$ de Selco para cada 1 milhão de rotíferos. Previamente à adição nos tanques de larvicultura, os rotíferos foram concentrados em malha de $25 \mu \mathrm{m}$ e limpos em água salgada filtrada.

Cistos de Artemia sp. (Great Salt Lake, Inve ${ }^{\circledR}$, Estados Unidos) foram eclodidos em tanques de fibra 
cilíndrico-cônicos, com volume útil de $35 \mathrm{~L}$ e aeração contínua durante $24 \mathrm{~h}$. A temperatura e a salinidade foram mantidas em $29^{\circ} \mathrm{C}$ e 30 , respectivamente. Para a eclosão manteve-se uma taxa de iluminação superior a 2000 lux. Os náuplios recém-eclodidos foram coletados em malha de $125 \mu \mathrm{m}$ e limpos com água salgada filtrada. Para o enriquecimento, foram estocados em tanques de $35 \mathrm{~L}$ (200.000 náuplios/L), onde receberam a emulsão comercial DC DHA Selco (Inve $^{\circledR}$, Estados Unidos) por $24 \mathrm{~h}$ em água na salinidade 30 e temperatura de $26 \stackrel{\circ}{\circ}$. Os protocolos para cultivo de rotífero e enriquecimento da Artemia foram realizados de acordo com as instruções sugeridas pelo fabricante.

A análise da pigmentação dos juvenis de $P$. orbignyanus foi realizada no final do experimento. Foi realizada uma amostragem aleatória de 20 juvenis de cada tratamento, que foram submetidos a uma avaliação da pigmentação. A classificação da pigmentação foi realizada de acordo Reitan et al. (1994), sendo constituída da seguinte forma: (1) completamente pigmentados, (2) parcialmente pigmentados e (3) não pigmentados.
Os efeitos do enriquecimento da Artemia sobre o crescimento e a sobrevivência dos juvenis de linguado foram avaliados através do teste " $\mathrm{t}$ " de Student com nível de significância de 95\%. Os resultados do experimento estão expressos em média \pm erro padrão. Os dados em percentagem foram previamente transformados em arco-seno. As análises foram realizadas utilizando o "software" Statistica $7.0^{\circledR}$.

\section{RESULTADOS}

Os juvenis alimentados com Artemia enriquecida apresentaram sobrevivência significativamente superior $(P<0,05)$, quando comparados aos juvenis alimentados com náuplios recém-eclodidos de Artemia (Figura 1). A sobrevivência dos juvenis alimentados com Artemia enriquecida foi de $42 \pm 8 \%$, enquanto que a dos linguados alimentados com náuplios de Artemia foi de $20 \pm 3 \%$.

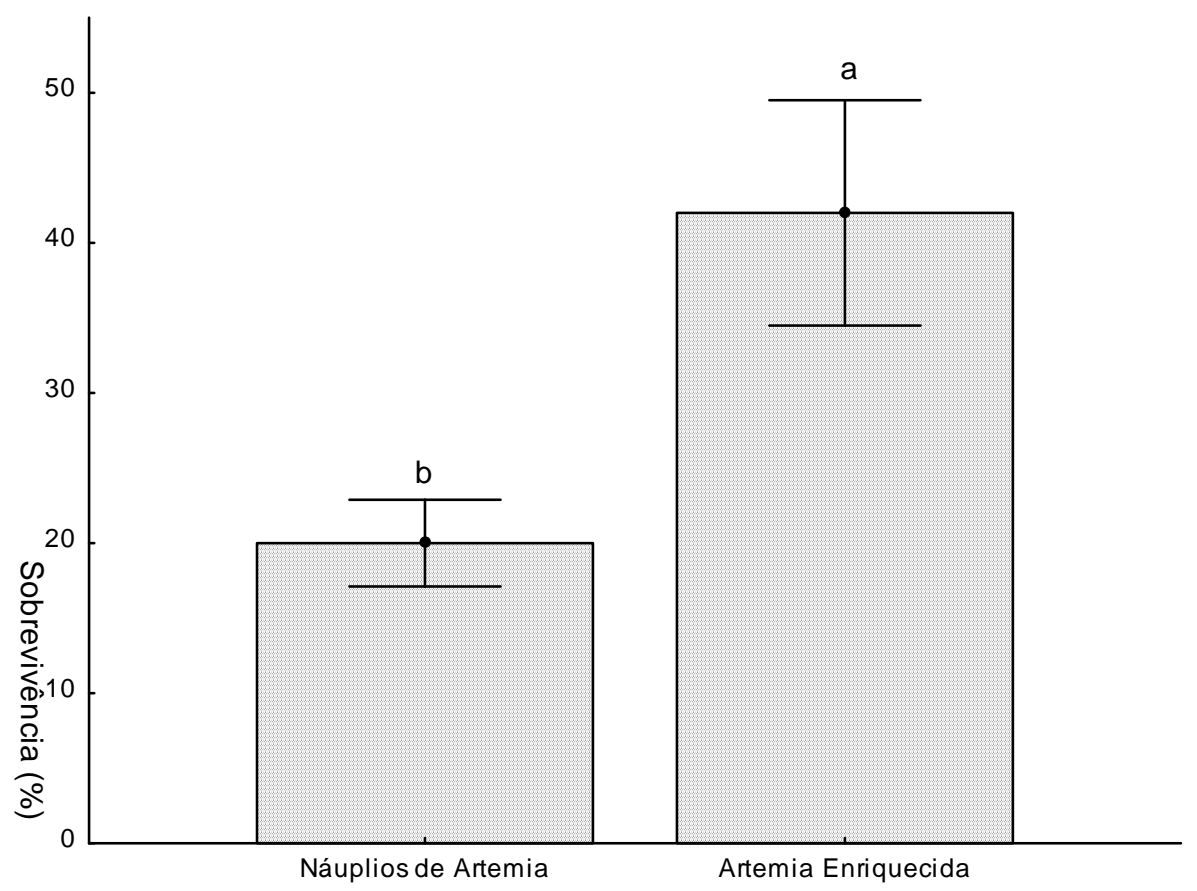

FIGURA 1 - Sobrevivência de juvenis do linguado Paralichthys orbignyanus alimentados com náuplios de Artemia ou Artemia enriquecida com n-3 HUFA. Letras diferentes sobre cada barra indicam diferença significativa $(P<0,05)$ entre os tratamentos. 
O crescimento dos juvenis alimentados com Artemia enriquecida, também foi significativamente superior $(P<0,05)$ ao dos juvenis alimentados com náuplios de Artemia (Figuras 2 e 3). Ao término do experimento o peso dos linguados alimentados com náuplios de Artemia foi $94,2 \pm 6,3 \mathrm{mg}$ enquanto que aqueles alimentados com Artemia enriquecida atingiram $154,9 \pm 10,3 \mathrm{mg}$. O comprimento total dos juvenis alimentados com náuplios de Artemia foi igual a $20,7 \pm 0,6 \mathrm{~mm}$ enquanto os alimentados com Artemia enriquecida atingiram $24,6 \pm 0,7 \mathrm{~mm}$.

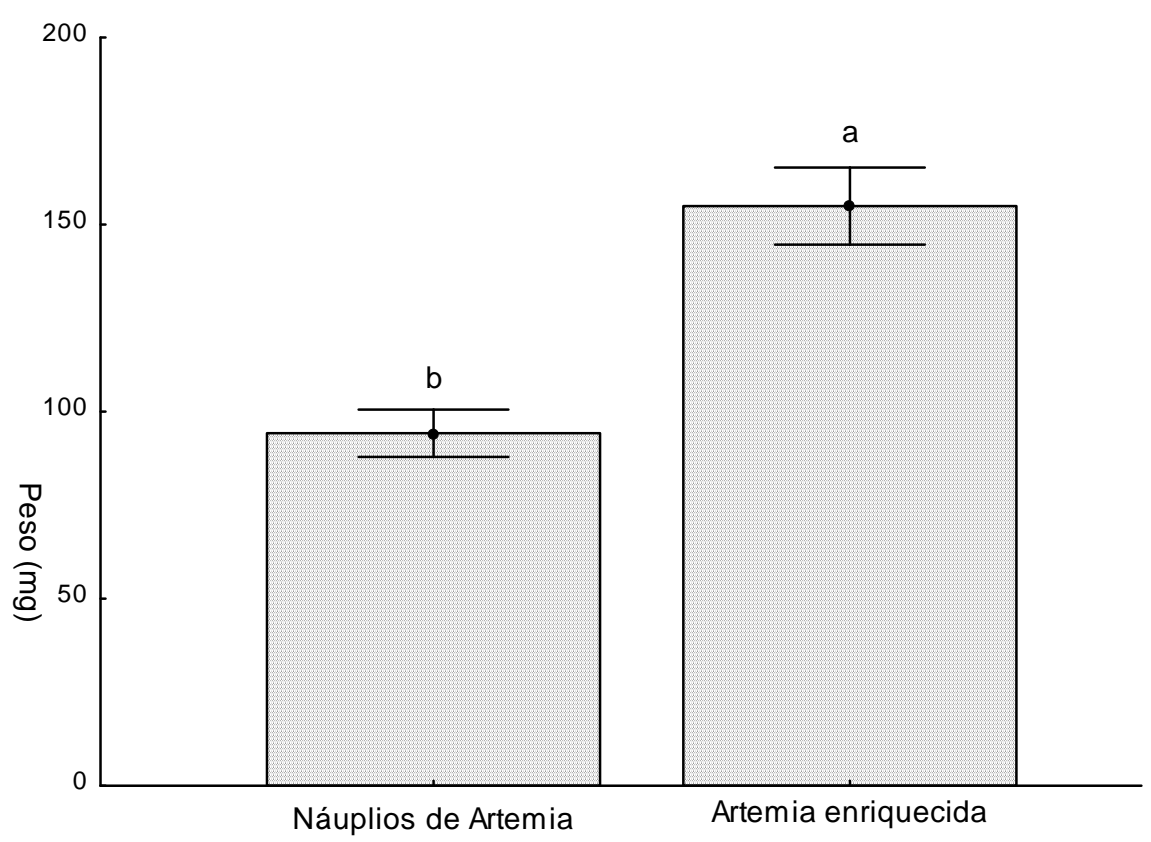

FIGURA 2 - Peso final de juvenis do linguado Paralichthys orbignyanus alimentados com náuplios de Artemia ou Artemia enriquecida com n-3 HUFA. Letras diferentes sobre cada barra indicam diferença significativa $(P<0,05)$ entre os tratamentos.

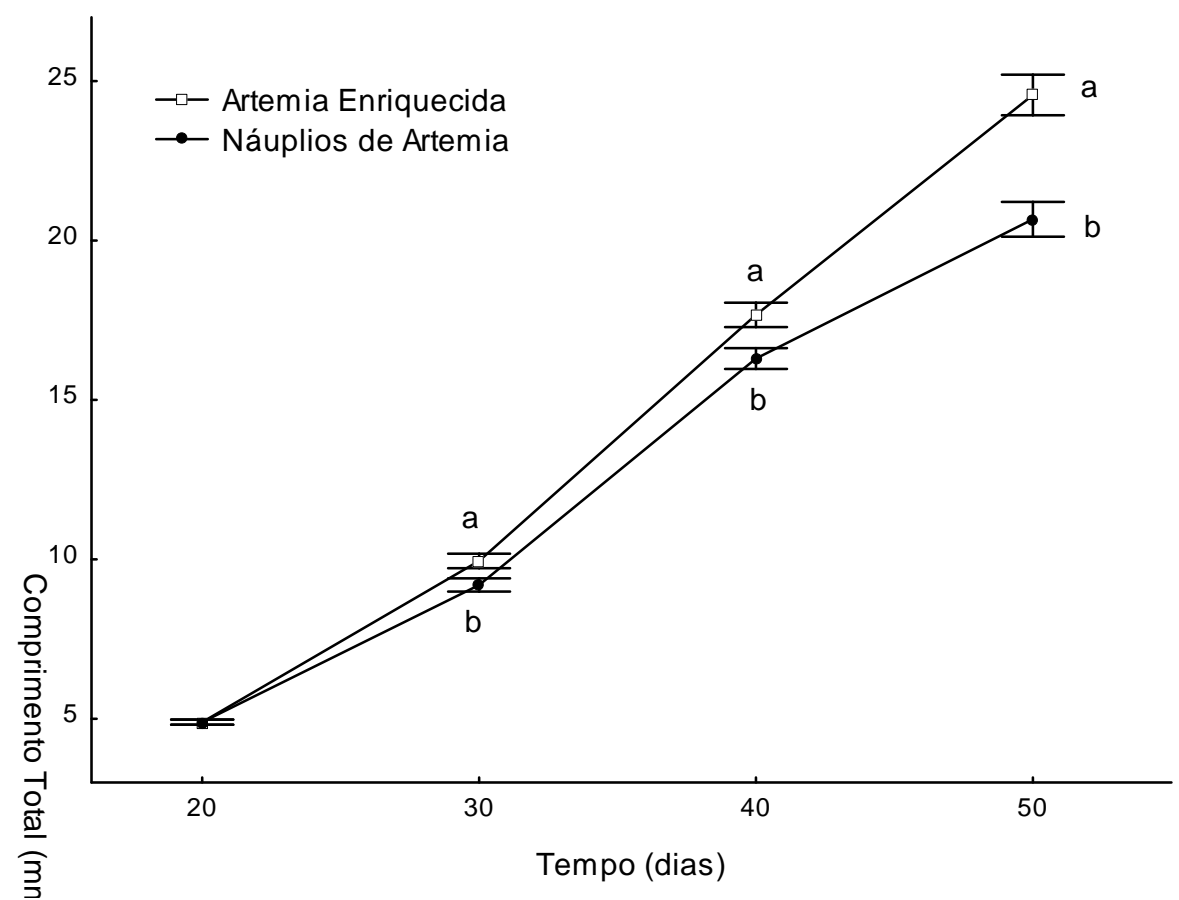

FIGURA 3 - Comprimento total de juvenis do linguado Paralichthys orbignyanus alimentados com náuplios recém-eclodidos de Artemia ou metanáuplios de Artemia enriquecidos com n-3 HUFA. Letras diferentes a cada tempo indicam diferença significativa $(P<0,05)$ entre os tratamentos. 
Quanto à pigmentação, os resultados foram semelhantes entre os tratamentos. Foi encontrado um maior percentual de linguados com pigmentação parcial, sendo $60 \%$ e $55 \%$ para os linguados alimentados com náuplios de Artemia e Artemia enriquecida, respectivamente. Os resultados demonstraram também que o percentual de juvenis com pigmentação completa foi de $20 \%$ para os linguados alimentados com náplios de Artemia e 25\% para os alimentados com Artemia enriquecida. Enquanto que para os juvenis totalmente desprovidos de pigmentação foi observada uma equidade de $20 \%$, entre os tratamentos.

\section{DISCUSSÃo}

Observou-se que juvenis de $P$. orbignyanus alimentados com Artemia enriquecida com n-3 HUFA apresentaram taxa de sobrevivência superior aos alimentados com náuplios recém-eclodidos de Artemia sem a presença de n-3 HUFA (Figura 1). Furuita et al. (1999) analisando diferentes níveis de n3 HUFA no alimento vivo para larvas do linguado Paralichthys olivaceus, obtiveram taxa de sobrevivência e crescimento superiores, quando comparadas às larvas alimentadas com ácido oléico (18:1n-9), ou seja, com reduzidos níveis de n-3 HUFA. Resultados similares foram encontrados por Hamre \& Harboe (2008) que observaram uma sobrevivência elevada em juvenis do linguado Hippoglossus hippoglossus alimentados com Artemia enriquecida com n-3 HUFA. Estes resultados comprovaram a necessidade da utilização de dietas iniciais enriquecidas com n-3 HUFA para diferentes espécies de linguado, uma vez que espécies marinhas de peixes são incapazes de sintetizar os ácidos graxos de cadeia longa (n-3 HUFA) (Kanazawa, 1997), sendo, portanto, fundamental incluí-los na dieta através de uma fonte rica nesses ácidos graxos.

De uma forma geral os linguados ao permanecerem por longos períodos em dietas deficientes em n-3 HUFA apresentam altas taxas de mortalidade, como foi o caso do "turbot" $P$. maxima (Bell et al. 1985) e do linguado Limanda ferruginea (Copeman et al. 2002).
Os melhores resultados de crescimento também foram observados para os linguados alimentados com Artemia enriquecida n-3 HUFA, quando comparado com a utilização de náuplios de Artemia recém-eclodidos (Figuras 2 e 3 ). Para os linguados $P$. dentatus (Baker et al. 1998), Paralichthys olivaceus (Furuita et al. 1998) e H. hippoglossus (Hamre \& Harboe 2008) e outras espécies de peixes como o "Korean rockfish" Sebastes schlegeli (Cho et al. 2001) e o bijupirá Rachycentron canadum (Faulk \& Holt, 2005) O enriquecimento do alimento vivo com n-3 HUFA também proporcionou melhor crescimento. $O$ menor crescimento observado nos juvenis alimentadas com Artemia não enriquecida demonstram uma vez mais que esses ácidos graxos são essenciais para o bom desenvolvimento de juvenis do $P$. orbignyanus criados em cativeiro.

A importância dos ácidos graxos n-3 HUFA como componentes da dieta para o aumento do sucesso da pigmentação tem sido relatada para várias espécies de linguados (Kanazawa et al. 1991; Baker et al. 1998; Estévez et al. 1999; Bolker \& Hill 2000). Entretanto, os índices de albinismo são muito variáveis entre diferentes espécies de linguado. $O$ percentual de albinismo encontrado (20\%) no presente estudo encontra-se dentro da faixa descrita para outras espécies de linguado. Reitan et al. (1994), por exemplo, encontraram taxas inferiores a $10 \%$ de albinismo para $P$. maxima, enquanto que para Limanda ferruginea este índice foi de 34\% (Purchase et al. 2002).

A similaridade entre os níveis de pigmentação encontrados neste trabalho para os linguados alimentados com ou sem suplementação de n-3 HUFA pode ser parcialmente explicada pelo manejo alimentar. Os rotíferos utilizados na alimentação das larvas de linguado neste experimento não foram enriquecidos com n-3 HUFA, portanto os linguados receberam alimento enriquecido somente após o final da metamorfose. Desse modo, é possível que o período crítico para pigmentação normal de $P$. orbignyanus ocorra antes da metamorfose, o que já foi descrito para outras espécies de linguado (Kanazawa 1991; Reitan et al. 1994; Copeman et al. 2002). 
Por outro lado, os parâmetros ambientais empregados durante este experimento podem ter influenciado a pigmentação dos linguados. Venizelos \& Benetti (1999) reportaram que a intensidade luminosa inadequada durante a produção de diferentes espécies de linguado pode afetar a pigmentação dos peixes. Nesse experimento foi utilizada uma intensidade luminosa de 100 lux durante $24 \mathrm{~h}$ por dia. Contudo a intensidade luminosa adequada para esta espécie ainda não foi determinada.

Portanto, para que sejam produzidos linguados bem pigmentados é muito importante definir com precisão o momento exato do fornecimento do alimento enriquecido para $P$. orbignyanus. Além disso, o papel da intensidade luminosa na pigmentação sobre esta espécie deve ser mais aprofundado através de novos estudos com vistas a esclarecer os diversos pontos ainda pouco conhecidos.

\section{CONCLUSÃO}

Os resultados desse trabalho mostram, que existe efeito benéfico do enriquecimento da Artemia com ácidos graxos altamente insaturados sobre a sobrevivência e o crescimento dos juvenis de $P$. orbignanus. Dessa forma 0 uso da Artemia enriquecida com n-3 HUFA deve ser empregado para que juvenis de melhor qualidade sejam produzidos.

\section{AGRADECIMENTOS}

Os autores agradecem ao Ministério da Pesca e Aqüicultura (MPA) e ao CNPq pelo financiamento deste trabalho. Ricardo V. Rodrigues é bolsista DTI 2 do CNPq e Luís André Sampaio é bolsista de produtividade em pesquisa do CNPq (308013/2009-3).

\section{REFERÊNCIAS}

ANDO, Y; H SAMOTO \& Y MURAYAMA. 2004. Positional distribution of DHA and EPA in tryacil-sn-glycerols (TAG) of Artemia franciscana nauplii enriched with fish oils ethyl esters and TAG. Aquaculture, 233: 321-335.

BAKER, EP; D ALVES \& DA BENGTSON. 1998. Effect of rotifer and Artemia fatty-acid enrichment on survival, growth and pigmentation of summer flounder Paralichthys dentatus larvae. J. World Aquacult. Soc., 29: 494-498.

BELL, MV; RJ HENDERSON; BJS PIRIE \& JR SARGENT. 1985. Effects of dietary polyunsaturated fatty acid deficiencies on mortality, growth and gill structure in the turbot, Scophthalmus maximus. J. Fish Biol., 26: 181-191.

BIANCHINI, A; W WASIELESKY JR \& KC MIRANDA-FILHO. 1996. Toxicity of nitrogenous compounds to juveniles of flatfish Paralichthys orbignyanus. Bull. Environ. Contam. Toxicol., 56: 453-459.

BIANCHINI, A; LA SAMPAIO \& RB ROBALDO. 2010. Cultivo de linguado Paralichthys orbignyanus. In: BALDISSEROTTO, B \& LC GOMES. (eds.). Espécies nativas para piscicultura no Brasil. Editora UFSM, $2^{\underline{a}}$ edição, Santa Maria, Brasil. Cap. 22: 559-587

BOLKER, JA \& CR HILL. 2000. Pigmentation development in hatchery-reared flatfishes. J. Fish Biol., 56: 1029-1052.

CHO, SH; SB HUR \& J-Y JO. 2001. Effect of enriched live feeds on survival and growth rates in larval Korean rockfish, Sebastes schlegeli Hilgendorf. Aquacult. Res., 32: 199-208.

COPEMAN, LA; CC PARRISH; JA BROWN \& M HAREL. 2002. Effect of docosahexaenoic, eicosapentaenoic, and arachidonic acids on the early growth, survival, lipid composition and pigmentation of yellowtail flounder (Limanda ferruginea): a live food enrichment experiment. Aquaculture, 210: 285-304.

ESTÉVEZ, A, LA MCEVOY; JG BELL \& JR SARGENT. 1999. Growth, survival, lipid composition and pigmentation of turbot (Scophthalmus maximus) larvae fed live-prey enriched in Arachidonic and Eicosapentaenoic acids. Aquaculture, 180: 321-343.

FAULK, CK \& GJ HOLT. 2005. Advances in rearing cobia Rachycentron canadum larvae in recirculating aquaculture systems: live prey enrichment and greenwater culture. Aquaculture, 249: 231-243.

FURUITA, H; T TAKEUCHI \& K UEMATSU. 1998. Effects of eicosapentaenoic and docosahexaenoic acids on growth, survival and brain development of larval Japanese flounder (Paralichthys olivaceus). Aquaculture, 161: 269-279.

FURUITA, H; K KONISHI \& T TAKEUCHI. 1999. Effect of different levels of eicosapentaenoic acid and docosahexaenoic acid in Artemia nauplii on growth, survival and salinity tolerance of larvae of Japanese flounder, Paralichthys olivaceus. Aquaculture, 170: 59-69.

HAMRE, K \& T HARBOE. 2008. Artemia enriched with high n-3 HUFA may give a large improvement in performance of Atlantic halibut (Hippoglossus hippoglossus L.) larvae. Aquaculture, 277: 239-243.

KANAZAWA, A. 1991. Nutritional mechanisms causing abnormal pigmentation in cultured marbled solea larvae, Limanda yokohamae (Heterosomata). In: LAVENS, P; P SORGELOOS, E JASPERS \& F OLIVIER. (eds.). Larvi'91, Fish and Crustacean Larviculture Symposium. Gent, Belgium, Special Publication: 20-22.

KANAZAWA, A. 1997. Effect of docosahexaenoic acid and phospholipids on stress tolerance of fish. Aquaculture, 155: 129-134.

LEGÉR, P; DA BENGSTON; KL SIMPSON \& P SORGELOOS. 1986. The use and nutritional values of Artemia as a food source. Oceanogr. Mar. Biol., 24: 521-623.

LEMA, T; MFT OLIVEIRA \& CAS LUCENA. 1980. Levantamento preliminar dos Pleuronectiformes do extremo sul do Brasil ao Rio de la Plata. Iheringia, 56: 25-52. 
MORAIS, S; L NARCISO; E DORES \& P POUSÃO-FERREIRA. 2004. Lipid enrichment for (Solea senegalensis) larvae: effect on larval growth, survival and fatty acid profile. Aquacult. Int., 12: 281-298.

PURCHASE, CF; DL BOYCE \& JA BROWN. 2002. Occurrence of hypomelanization in cultured yellowtail flounder Limanda ferruginea. Aquacult. Res., 33: 1191-1193.

REITAN, IK; JR RAINUZZO \& Y OLSEN. 1994. Influence of lipid composition of live feed on growth, survival and pigmentation of turbot larvae. Aquacult. Int., 2: 33-48.

SAMPAIO, LA \& A BIANCHINI. 2002. Salinity effects on osmoregulation and growth of the euryhaline flounder Paralichthys orbignyanus. J. Exp. Mar. Biol. Ecol., 269: 187196.

SAMPAIO, LA; A BIANCHINI \& VR CERQUEIRA. 2001. Growth of juvenile Brazilian flounder, Paralichthys orbignyanus, cultured at different salinities. J. Appl. Aquacult., 11: 67-75.

SAMPAIO, LA; LS FREITAS; MH OKAMOTO; LR LOUZADA; RV RODRIGUES \& RB ROBALDO. 2007. Effects of salinity on Brazilian flounder Paralichthys orbignyanus from fertilization to juvenile settlement. Aquaculture, 262: 340-346.

SAMPAIO, LA; RB ROBALDO \& A BIANCHINI. 2008. Hormone induced ovulation, natural spawning and larviculture of Brazilian flounder Paralichthys orbignyanus._Aquacult. Res., 39: 712-717.

SARGENT, JR. 1995. Origins and functions of egg lipids. In: BROMAGE, NR \& RJ ROBERTS. (eds.). Broodstock
Management and Egg and Larval Quality. Blackwell, Oxford, Inglaterra, Chap. 14: 353-372.

SORGELOOS, P; P COUTTEAU; P DHERT; G MERCHIE \& $P$ LAVENS. 1998. Use of brine shrimp, Artemia spp., in larval crustacean nutrition: a review. Rev. Fish. Sci., 6: 55-68.

SORGELOOS, P; P DHERT \& P CANDREVA. 2001. Use of brine shrimp, Artemia spp., in marine fish larviculture. Aquaculture, 200: 147-159.

VENIZELOS, A \& D BENETTI. 1999. Pigment abnormalities in flatfish. Aquaculture, 176: 181-188.

WASIELESKY JR, W, A BIANCHINI, MH SANTOS \& LH POERSCH. 1997. Tolerance of juvenile flatfish Paralichthys orbignyanus to acid stress. J. World Aquacult. Soc., 28: 202204.

WASIELESKY JR, W, K MIRANDA-FILHO \& A BIANCHINI. 1998. Tolerancia a la temperatura de juveniles de lenguado Paralichthys orbignyanus. Frente Marít., 17: 43-48.

WATANABE, T, C KITAJIMA \& S FUJITA. 1983. Nutritional values of live food organisms used in Japan for mass propagation of fish: a review. Aquaculture, 34: 115-143.

Submetido $-21 / 12 / 2011$

Aceito - 06/03/2012 
